\title{
Spindly attachments
}

\author{
Filiz Çivril $^{1}$ and Andrea Musacchio ${ }^{1,2,3}$ \\ ${ }^{1}$ Department of Experimental Oncology, European Institute of Oncology, I-20139 Milan, Italy; ${ }^{2}$ Research Unit of the Italian \\ Institute of Technology (IIT) Foundation at the IFOM-IEO Campus, I-20139 Milan, Italy
}

\begin{abstract}
The attachment of chromosomes to spindle microtubules during mitosis is a delicate and intricate process on which eukaryotic cells critically depend to maintain their ploidy. In this issue of Genes \& Development, Gassmann and colleagues (pp. 2385-2399) present an analysis of the recently discovered Spindly/SPDL-1 protein that casts new lights onto the attachment process and the way it relates to the control of cell cycle progression.
\end{abstract}

\section{The basics}

Mitotic prometaphase is quite an eventful phase of the eukaryotic cell cycle. Its most characteristic trait is the mitotic spindle's frantic engagement in the capture of replicated chromosomes (the sister chromatids) that have been scattered throughout the cell. Microtubules, the main ingredient of the mitotic spindle, form tight attachments with specialized structures on mitotic chromosomes known as kinetochores. The correct configuration of kinetochore-microtubule attachment is named biorientation (Fig. 1A). When bioriented, the chromatids in a sister chromatid pair in the mother cell are connected to opposite spindle poles (Cheeseman and Desai 2008; Tanaka and Desai 2008). Biorientation contributes to chromosome congression to the metaphase plate. It also ensures that when the cohesion linking the sisters is removed at the metaphase-to-anaphase transition, the sisters are separated toward opposite spindle poles to give rise to two daughter cells with identical genetic material (Fig. 1B).

\section{The kinetochore}

The 60-80 conserved proteins that populate mitotic kinetochores from yeast to humans can be schematically subdivided into distinct functional modules (for review, see Cheeseman and Desai 2008). The first module is implicated in the interaction of kinetochores with centromeric chromatin, and is built around a specialized nucleosome containing the histone $\mathrm{H} 3$ variant CENP-A

[Keywords: Centromere; aneuploidy; mitosis; kinetochore; microtubule; spindle; chromosome]

${ }^{3}$ Corresponding author.

E-MAIL andrea.musacchio@ifom-ieo-campus.it; FAX 39-02-57489851. Article is online at http://www.genesdev.org/cgi/doi/10.1101/gad.1719208. and associated binding partners in the so-called constitutive centromere-associated network (CCAN; also known as NAC/CAD) (Cheeseman and Desai 2008). The second module provides the core of the microtubulebinding interface. Its most prominent component is the KNL1-Mis12-Ndc80 complex (KMN) network, an array of 10 proteins (Cheeseman and Desai 2008). Besides creating a receptor for the microtubule, the KMN network also serves as a recruitment pad for additional proteins, including molecular motors like dynein, which have been implicated in the early stages of attachment (see below). Although the points of contact between the first and second modules have not been elucidated, the two modules contribute a structural core of the kinetochore that physically links chromosomes to spindle microtubules (Fig. 1A).

The additional modules are implicated in the control of the state of kinetochore-microtubule attachment. One module includes the components of the spindle assembly checkpoint (SAC), which are all recruited to kinetochores in mitosis (Musacchio and Salmon 2007). The SAC alleviates the potentially hazardous consequences of attachment being largely (although not solely) based on the random encounter of a kinetochore with a microtubule (Cheeseman and Desai 2008). Within a single cell, there can be substantial variability in the timing at which the different chromosomes biorient (for example, see Meraldi et al. 2004). Conversely, the loss of sister chromatid cohesion is always highly synchronous, and it always follows biorientation of the last sister chromatid pair. The synchronization is due to the SAC (for review, see Musacchio and Salmon 2007). As long as there is even a single unattached chromosome in the mitotic cell, the SAC continues to generate a "wait anaphase" signal to prevent loss of sister chromatid cohesion and mitotic exit. Once all chromosomes are properly attached, the SAC signal subsides, sister chromatid cohesion is removed, and the sisters are finally separated into daughter cells that are being concomitantly created through the mitotic exit program (Musacchio and Salmon 2007).

Through yet another module, kinetochores have also acquired the remarkable ability to regulate the stability of microtubule attachments, and to recognize and correct attachments that fail to result in biorientation. A crucial piece of machinery in the control of attachment stability is the chromosome-passenger complex, whose 

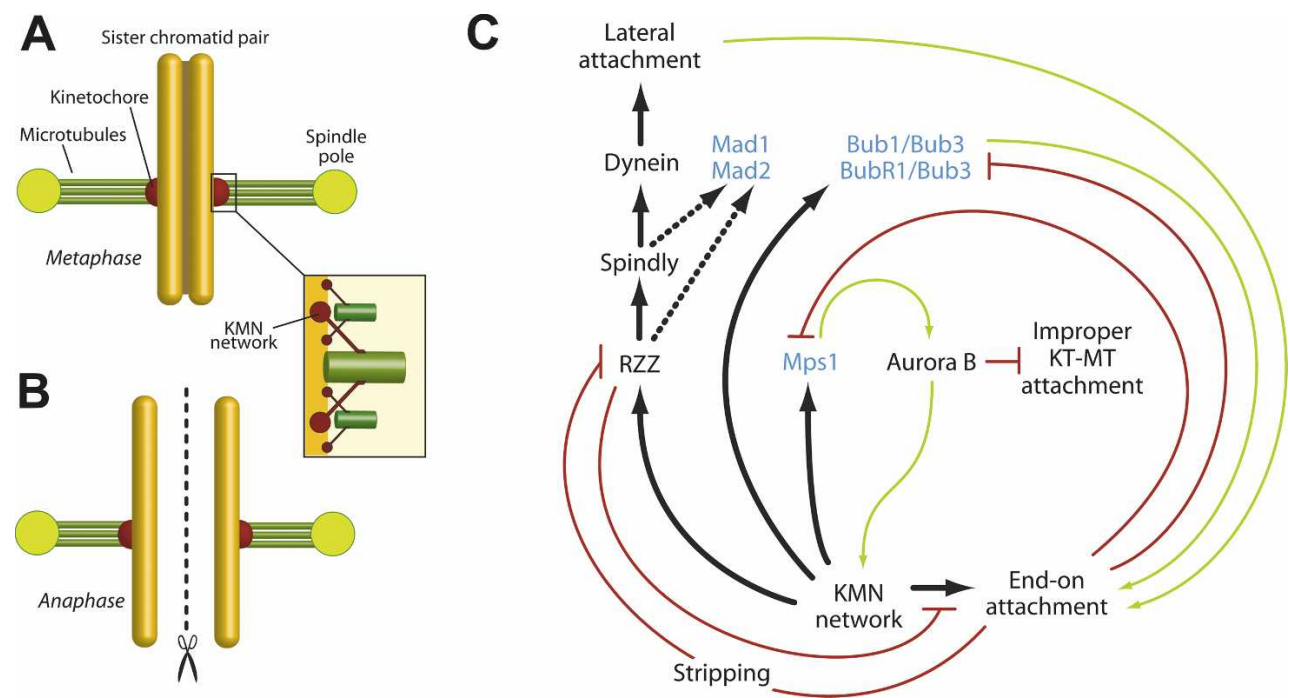

Figure 1. Complexity of the dynamic kinetochore-microtubule interface. $(A)$ The sister kinetochores of a bioriented sister chromatid pair at metaphase are connected to opposite spindle poles through one or more microtubules (the number of microtubules varies in different species). The closeup shows the configuration of end-on attachment with KMN networks bound to microtubules. (B) At anaphase, the sister chromatids have lost cohesion and are transported to opposite spindle poles. $(C)$ Complexity of the kinetochoremicrotubule attachment process. Black arrows indicate an action, such as "recruitment" or "microtubule binding." Dotted black arrows indicate that an uncertainty exists on the actual species performing the action. Green arrows define positive regulation. Red lines with a smaller perpendicular line at one end indicate negative regulation. We did not distinguish whether the negative or positive regulation is exercised on an action or the species that carries out the action. The KMN network mediates several actions, including kinetochore recruitment of the RZZ complex and of the SAC proteins Mps1, Bub1, BubR1, and Bub3, and the formation of stable end-on microtubule attachments. Most and probably all of the actions ascribed to the KMN network are positively regulated by the Aurora B kinase. Aurora B also suppresses improper kinetochore-microtubule attachments. The checkpoint proteins (in blue) positively regulate the process of end-on attachment. In the case of $\mathrm{Mps1}$, this may occur through a positive regulation of Aurora B activity. In the case of Bub1 and BubR1, the target of regulation by these kinases is unknown. After recruitment to prometaphase kinetochores, the RZZ complex suppresses the ability of the KMN network to bind to microtubules. Once at kinetochore, the RZZ complex recruits Spindly, dynein, Mad1, and Mad2. Dynein mediates lateral attachment to microtubules, which in turn favors end-on attachment. The establishment of end-on attachment suppresses the kinetochore accumulation of the RZZ and of the other proteins whose kinetochore localization depends on the RZZ, as it instates the mechanism of "stripping." See the text for details and references supporting this scheme.

most renowned component is the Aurora B kinase (Ruchaud et al. 2007).

If a modular subdivision is a useful aid to grasp the basic functions of kinetochores, current analyses have to address the molecular complexity of kinetochores, in particular, the way in which different modules control each other dynamically. Elements of this regulation are continuing to emerge. For instance, it is now clear that the main piece of microtubule attachment machinery, the KMN network, is responsible for the recruitment and release of the SAC components to/from kinetochores, and that the Aurora B kinase phosphorylates KMN network components to regulate their binding affinity for microtubules and their ability to recruit the SAC (Fig. 1C; Musacchio and Salmon 2007; Cheeseman and Desai 2008). These examples, which will be analyzed more thoroughly below, are forcing a shift of paradigm in the way we think about the dynamic kinetochore-microtubule interface.

\section{The molecular machinery of kinetochore-microtubule attachment}

On their route toward achieving biorientation, kinetochores go through two distinct types of microtubule at- tachment (Tanaka and Desai 2008). Early during the attachment process, kinetochores interact laterally with microtubules, and in many species this initial attachment results in rapid poleward movement of the attached chromosome (see below). At later stages, however, kinetochores become engaged in so-called end-on attachments, in which the kinetochores are tethered to the microtubule plus end through a "sliding collar" whose motility is processively coupled to the polymerization and depolymerization of kinetochore microtubules (Tanaka and Desai 2008). The formation of end-on attachments is critical for biorientation and for the generation of load bearing attachments. It is also necessary for the decline of the SAC signal prior to anaphase (Musacchio and Salmon 2007).

Recent work from several laboratories supports the conclusion that the components of the KMN network contribute the main interface for microtubule end-on attachment (for review, see Cheeseman and Desai 2008). As explained above, the KMN network takes its name from its components, which include KNL-1 (also known as Spc105 or blinkin, and which engages in a dimeric complex with Zwint-1/Ydr532p), the four-protein Mis12/Mtw1 complex (also containing Nnf1, Nsl1, and 
Dsn1), and the four-protein Ndc80 complex (also containing Nuf2, Spc24, and Spc25). The composition of the KMN network is conserved in different species (Cheeseman and Desai 2008). Two subunits in the Ndc80 complex, Ndc80 and Nuf2, contain calponin homology $(\mathrm{CH})$ domains in a tight dimeric arrangement that defines a single microtubule-binding interface (Wei et al. 2007; Ciferri et al. 2008). The binding affinity for microtubules increases when the Ndc80 complex is embedded in the KMN network (Cheeseman et al. 2006), possibly as a consequence of the formation of an oligomeric structure that involves multiple copies of each of the constituents of the KMN network (Joglekar et al. 2006).

While the KMN network is essential for end-on kinetochore-microtubule attachment, molecular motors play an important function in the initial lateral attachment of kinetochores to microtubules and in subsequent movements of chromosomes along microtubules. In budding yeast, Kar3, a member of the minus-end-directed kinesin 14 family, has been implicated in the rapid sliding of kinetochores on the microtubule lattice that precedes end-on attachment (Tanaka et al. 2007). In metazoans, the impairment of Ndc80 prevents load-bearing attachments but does not affect pole-directed prometaphase chromosome movements (Desai et al. 2003; Vorozhko et al. 2008). Newly captured "mono-oriented" chromosomes move toward the pole with velocities that can exceed $50 \mu \mathrm{m} / \mathrm{min}$, suggesting an involvement of dynein, another minus-end-directed motor (Rieder and Alexander 1990; King et al. 2000; Yang et al. 2007; Vorozhko et al. 2008). Indeed, the concomitant depletion of the Ndc80 complex and of dynein results in a "kinetochore-null" phenotype in which mitotic chromosomes are devoid of any motility (Desai et al. 2003; Vorozhko et al. 2008).

\section{Spindly, a novel player in the attachment saga}

The mechanism of maturation of a lateral attachment into an end-on attachment is poorly understood. As dynein is implicated in the initial attachment of chromosomes to microtubules, it is important to investigate the effects of inhibiting dynein onto the ability of chromosomes to achieve end-on attachment. Until recently, the functional analysis of kinetochore dynein has been complicated by the multiple roles of dynein in spindle formation (Banks and Heald 2001). The discovery that a tight complex of the three proteins Rough deal (Rod), Zeste-white 10 (ZW10), and Zwilch, abbreviated as the RZZ complex (Karess 2005), is responsible for recruiting dynein to mitotic kinetochores provided a new means to interfere specifically with kinetochore dynein (Starr et al. 1998).

If dynein recruitment to kinetochores is prevented by RNAi-mediated ablation of ZW10, proper chromosome congression and the formation of load-bearing attachments are moderately but significantly delayed, and lagging chromosomes are observed at anaphase, likely a consequence of merotelic attachments, incorrect attachments where a kinetochore becomes attached to opposite spindle poles (Karess and Glover 1989; Williams et al. 1992; Savoian et al. 2000; Buffin et al. 2007; Li et al. 2007; Yang et al. 2007). This phenotype has led to suggest that kinetochore dynein might not only be responsible for the rapid poleward movement of mono-oriented kinetochores, but also for the acceleration of the formation of stable end-on attachment required for biorientation. Other analyses, however, have suggested that the formation of a metaphase plate is unaffected when dynein is inhibited by means other than RZZ inhibition (Howell et al. 2001; Vorozhko et al. 2008).

A possible explanation for the discrepancy is that the effects of inhibiting ZW10 and its binding partners in the RZZ complex, rather than being limited to dynein mislocalization, also extend to an as yet unknown aspect of the microtubule attachment process mediated by the RZZ. The explanation has now gained considerable weight after two recent reports described the function of a novel protein, named Spindly, as a kinetochore-specific regulator of dynein (Griffis et al. 2007; Gassmann et al. 2008). Spindly was originally identified in a screen in Drosophilia melanogaster S2 cells as a protein whose ablation causes a mitotic arrest and changes in cell morphology (Griffis et al. 2007). It must be mentioned that the name Spindly has also been attributed to an unrelated protein involved in gibberellin signal transduction in Arabidopsis thaliana. Here, however, we will use the name Spindly exclusively to refer to CG15415 and its putative homologues in different species. Drosophila $(\mathrm{Dm})$ Spindly is an 807-residue protein characterized by the presence of a predicted coiled-coil-rich region in its N-terminal half (Griffis et al. 2007). Despite low sequence conservation and significantly different overall sizes, human (605 residues) and Caenorhabditis elegans (479 residues) homologs of Spindly, named HsSpindly and SPDL-1, respectively, were identified based on the overall structural conservation of the coiled-coil region and on a very conserved motif in the N-terminal half of the protein (Griffis et al. 2007; Gassmann et al. 2008).

\section{The Spindly phenotype}

Spindly, which relies on the RZZ complex for its own kinetochore recruitment, acts in turn as a kinetochore recruitment factor for dynein (Griffis et al. 2007; Gassmann et al. 2008). In their elegant analysis of Spindly (SPDL-1) in the C. elegans embryo, Gassmann et al. (2008) found that the depletion of Spindly results in a dramatic chromosome segregation defect, caused by the inability to form load-bearing attachments until just prior to anaphase. As Spindly and dynein are recruited to kinetochores by the RZZ complex, these investigators asked whether the phenotype when removing the RZZ complex would recapitulate the removal of Spindly. In agreement with previous observations, but somewhat surprisingly, they observed that rather than preventing the formation of load-bearing attachments, the depletion of RZZ subunits only resulted in a slight delay in the formation of load-bearing attachments and in a modest increase in chromosome segregation errors at anaphase |Griffis et al. 2007; Li et al. 2007; Yang et al. 2007; Gass- 
mann et al. 2008). Thus, in spite of a requirement on the RZZ to recruit Spindly to kinetochores, the phenotype when ablating Spindly in C. elegans is much more dramatic than the phenotype when ablating RZZ subunits in this organism.

A reasonable hypothesis put forward by Gassmann et al. (2008) to explain the difference of the Spindly and RZZ phenotypes is that the RZZ complex acts as a transient inhibitor of the KMN complex, and that Spindly modulates this function of the RZZ in a dynein-dependent manner. The investigators propose a working model in which Spindly monitors the formation of lateral microtubule attachments by dynein. Upon successful contact with microtubules, Spindly contributes to relieving the inhibitory role of the RZZ on the KMN network, thus promoting the maturation of lateral attachments into end-on attachments. In the absence of Spindly, the attachment restraining function of the RZZ onto the KMN network cannot be turned off until just prior to anaphase and the timing of formation of strong end-on attachments is compromised. Conversely, in the absence of the attachment restraining function of the RZZ, kinetochores can form stable end-on attachments, but they do so precipitously, becoming prone to form faulty attachments that might result in the appearance of lagging chromosomes at anaphase.

The generality of the model proposed in the previous paragraph to explain the relationship between Spindly, the RZZ, and dynein will have to be investigated by targeting the RZZ and Spindly in other organisms and cell types. As Spindly and the RZZ only exist in metazoans, their functions must be limited to the organisms that contain them, but their relevance may vary in different organisms. For instance, the ablation of Spindly in flies only delays the formation of a metaphase plate, but does not alter the formation of load-bearing attachments, and does not cause severe chromosome segregation problems (Griffis et al. 2007). Additional details from the phenotypes when ablating Spindly in flies or worms do not coincide. For instance, Dm Spindly is dispensable for kinetochore recruitment of dynactin (a dynein-associated complex) and of the SAC protein Mad2 (Griffis et al. 2007), while SPDL-1 appears to be essential for kinetochore recruitment of both these proteins (Gassmann et al. 2008). The origin of these differences is currently unclear.

Nevertheless, a conclusion that seems to be granted based on the studies of Gassmann et al. (2008) is that the role of kinetochore dynein is not limited to the poledirected movement of chromosomes, but extends to the control of the maturation of kinetochore-microtubule attachment. Furthermore, the discovery of a direct role of the RZZ complex on kinetochore-microtubule attachment argues that the ablation of the RZZ might not be the most direct way of testing the importance of kinetochore dynein in kinetochore-microtubule attachment.

\section{Dynamic control of kinetochore function}

Future studies will have to unveil the molecular bases of the influence of Spindly, dynein, and the RZZ on KMN function. From a more distant point of observation, the study of Spindly also depicts a new exciting connection between kinetochore modules. To clarify this point, it is important to remind the readers that besides contributing to the recruitment of dynein, the RZZ has been shown to be important for kinetochore recruitment of critical components of the SAC, such as Mad1 and Mad2, explaining the well-established requirements of the RZZ for SAC function (Basto et al. 2000; Chan et al. 2000; Buffin et al. 2005; Kops et al. 2005). By recruiting dynein to kinetochores, the RZZ also contributes to the downregulation of the SAC. Using kinetochore-bound microtubules as tracks, the dynein engine "strips" several SAC proteins from kinetochores, including Mad1, Mad2, and the components of the RZZ, and creates a stream of these proteins toward the spindle pole (Howell et al. 2001; Wojcik et al. 2001; Basto et al. 2004). Thus, the $\mathrm{RZZ}$ is responsible for dual control over the SAC: It promotes kinetochore recruitment of the SAC, but it also promotes kinetochore recruitment of dynein, which is implicated in down-regulation of the SAC after microtubule capture, providing a nice example of feedback control in the interaction of the SAC and the attachment machinery (Fig. 1C).

It is useful to place this concept in the general context of the dynamic control of kinetochore function. As anticipated above, a key question in kinetochore biology concerns the way in which different kinetochore modules are integrated to provide an appropriate response to the status of attachment. Several recent observations have brought to light the remarkable intricacy of this relationship. In particular, a first generalization emerging from the recent literature is that the KMN network regulates the recruitment of all SAC components to the kinetochore /with the notable exception of the Aurora B kinase, whose precise role in the SAC remains controversial). For instance, building on the original observation that the Ndc80 complex is required to recruit Mad1, Mad2, and Mps1 to kinetochores (Martin-Lluesma et al. 2002), a recent important study clarified that Bub1, BubR1, and Bub3 are recruited to kinetochores through what appears to be a direct interaction with the KNL-1/ Spc105/Blinkin subunit of the KMN network (Kiyomitsu et al. 2007). Thus, the microtubule attachment machinery mediates kinetochore recruitment of the SAC, suggesting that one or more physical properties of the kinetochore-microtubule attachment site, of which the $\mathrm{KMN}$ is the main constituent, are dynamically monitored to promote the activation and inactivation of the SAC signal.

Even more intriguing is the discovery of a reverse relationship implicating the kinase components of the SAC in the control of the mechanism of microtubule attachment. RNAi-mediated ablation of the SAC kinases Bub1, BubR1, and Mps1 results in severe misalignment problems coming along with the overriding of the SAC response (Johnson et al. 2004; Lampson and Kapoor 2005; Meraldi and Sorger 2005; Maure et al. 2007; Jelluma et al. 2008). It is possible that the effects of the ablation of the SAC kinases on kinetochore-microtubule attachment 
reflect a role for these kinases that is independent from their involvement in the SAC. On the other hand, it is sensible to postulate that these kinases participate in a single complex molecular process, characterized by extensive feedback control whereby the SAC kinases contribute to the creation of sturdy kinetochore-microtubule attachments and are concomitantly negatively regulated by attachment, which is in itself a highly dynamic process. In a recent landmark paper, Jelluma et al. (2008) provided a vivid representation of these ideas. These investigators demonstrated that Mps1 phosphorylates borealin, one of the components of the chromosome passenger complex, and is so doing controls the catalytic output of Aurora B and its influence on the kinetochore-microtubule attachment process and on the SAC (Fig. 1C).

The take-home message from these studies is that the pathways regulating the response to unattached kinetochores are anything but linear, and that more elaborate models of kinetochore function that formalize this complexity are needed (Fig. 1C). The emerging picture is that the attachment machinery and the SAC are intricately connected. Gaining a precise understanding of the molecular bases of kinetochore-microtubule attachment and of the interactions with the SAC will help unravel this complexity. The properties of Spindly and of the RZZ complex can be boxed in the same conceptual framework provided by the examples above. The RZZ is recruited to kinetochores through an interaction with Zwint-1, one of the components of the KMN network (Karess 2005). By contributing to the recruitment of Mad1, Mad2, and dynein to kinetochores, and by controlling the microtubule-binding activity of the KMN network, Spindly and the RZZ position themselves at the crossroad between the attachment and SAC machineries, coordinating their functions in ways that remain poorly understood.

\section{Conclusions}

In summary, there are many elements to believe that to be brought to successful completion, the process of chromosome segregation requires the seamless integration of activity from the microtubule-binding and SAC machineries. The elucidation of the molecular bases of the dynamic integration of kinetochore functions is a key goal for future studies. Specifically, three fundamental questions that future studies will have to address concern (1) the mechanism of conversion from lateral to end-on attachment; (2) the mechanisms that allow kinetochores to distinguish between proper and improper attachments, allowing for the selective destabilization of the latter and for the retention and stabilization of the former; and (3) the regulation of the dynamic response of the SAC during the attachment process. A question that underlies all three problems is what is being sensed by the attachment and SAC machinery to generate a dynamic response during attachment. Answering this question will amount to find a solution to the eternal dualism of tension versus attachment that has domi- nated the kinetochore field for the last two decades. The available data suggest that the sensory mechanism is based on the complex interaction of Aurora B, the KMN network, the RZZ, dynein, the SAC, and, now that it has been uncovered, Spindly. For a better understanding, we need to build clearer views of the structural organization of kinetochores, because it is only through a better knowledge of how kinetochores are organized-the reciprocal localization of its components-that we can derive testable hypotheses of the mechanisms through which different modules integrate their functions. More details have yet to emerge from a picture that now appears to be significantly more complex than we could have imagined.

\section{Acknowledgments}

We apologize to all those authors whose work could not be cited due to space restrictions. We thank the members of the Musacchio laboratory for many helpful discussions. Work in the Musacchio laboratory is funded by the Association for International Cancer Resarch (AICR), the Telethon Foundation, the EU FP6 program contracts 3D-Repertoire and Mitocheck, the Italian Association for Cancer Research (AIRC), the Fondo di Investimento per la Ricerca di Base (FIRB), and the Italian Ministry of Health. F.C. is a graduate student of the European School of Molecular Medicine, and her fellowship is generously funded by the Nando Peretti Foundation.

\section{References}

Banks, J.D. and Heald, R. 2001. Chromosome movement: Dynein-out at the kinetochore. Curr. Biol. 11: R128-R131. doi: 10.1016/S0960-9822/01)00059-8.

Basto, R., Gomes, R., and Karess, R.E. 2000. Rough deal and $\mathrm{Zw} 10$ are required for the metaphase checkpoint in Drosophila. Nat. Cell Biol. 2: 939-943.

Basto, R., Scaerou, F., Mische, S., Wojcik, E., Lefebvre, C., Gomes, R., Hays, T., and Karess, R. 2004. In vivo dynamics of the rough deal checkpoint protein during Drosophila mitosis. Curr. Biol. 14: 56-61.

Buffin, E., Lefebvre, C., Huang, J., Gagou, M.E., and Karess, R.E. 2005. Recruitment of Mad2 to the kinetochore requires the Rod/Zw10 complex. Curr. Biol. 15: 856-861.

Buffin, E., Emre, D., and Karess, R.E. 2007. Flies without a spindle checkpoint. Nat. Cell Biol. 9: 565-572.

Chan, G.K., Jablonski, S.A., Starr, D.A., Goldberg, M.L., and Yen, T.J. 2000. Human Zw10 and ROD are mitotic checkpoint proteins that bind to kinetochores. Nat. Cell Biol. 2: 944-947.

Cheeseman, I.M. and Desai, A. 2008. Molecular architecture of the kinetochore-microtubule interface. Nat. Rev. Mol. Cell Biol. 9: 33-46.

Cheeseman, I.M., Chappie, J.S., Wilson-Kubalek, E.M., and Desai, A. 2006. The conserved KMN network constitutes the core microtubule-binding site of the kinetochore. Cell 127: 983-997.

Ciferri, C., Pasqualato, S., Screpanti, E., Varetti, G., Santaguida, S., Dosreis, G., Maiolica, A., Polka, J., Deluca, J., and Dewulf, P. 2008. Implications for kinetochore-microtubule attachment from the structure of an engineered Ndc80 complex. Cell 133: 427-439.

Desai, A., Rybina, S., Müller-Reichert, T., Shevchenko, A., Shevchenko, A., Hyman, A., and Oegema, K. 2003. KNL-1 
directs assembly of the microtubule-binding interface of the kinetochore in C. elegans. Genes \& Dev. 17: 2421-2435.

Gassmann, R., Essex, A., Hu, J.-S., Maddox, P.S., Motegi, F., Sugimoto, A., O'Rourke, S.M., Bowerman, B., McLeod, I., Yates III, J.R., et al. 2008. A new mechanism controlling kinetochore-microtubule interactions revealed by comparison of two dynein-targeting components: SPDL-1 and the Rod/Zwilch/Zw10 complex. Genes \& Dev. (this issue). doi: $10.1101 / \mathrm{gad} .1687508$.

Griffis, E.R., Stuurman, N., and Vale, R.D. 2007. Spindly, a novel protein essential for silencing the spindle assembly checkpoint, recruits dynein to the kinetochore. J. Cell Biol. 177: 1005-1015.

Howell, B.J., McEwen, B.F., Canman, J.C., Hoffman, D.B., Farrar, E.M., Rieder, C.L., and Salmon, E.D. 2001. Cytoplasmic dynein/dynactin drives kinetochore protein transport to the spindle poles and has a role in mitotic spindle checkpoint inactivation. J. Cell Biol. 155: 1159-1172.

Jelluma, N., Brenkman, A.B., van den Broek, N.J., Cruijsen, C.W., van Osch, M.H., Lens, S.M., Medema, R.H., and Kops, G.J. 2008. Mps1 phosphorylates Borealin to control Aurora B activity and chromosome alignment. Cell 132: 233-246.

Joglekar, A.P., Bouck, D.C., Molk, J.N., Bloom, K.S., and Salmon, E.D. 2006. Molecular architecture of a kinetochoremicrotubule attachment site. Nat. Cell Biol. 8: 581-585.

Johnson, V.L., Scott, M.I., Holt, S.V., Hussein, D., and Taylor, S.S. 2004. Bub1 is required for kinetochore localization of BubR1, Cenp-E, Cenp-F and Mad2, and chromosome congression. J. Cell Sci. 117: 1577-1589.

Karess, R. 2005. Rod-Zw10-Zwilch: A key player in the spindle checkpoint. Trends Cell Biol. 15: 386-392.

Karess, R.E. and Glover, D.M. 1989. rough deal: A gene required for proper mitotic segregation in Drosophila. J. Cell Biol. 109: 2951-2961.

King, J.M., Hays, T.S., and Nicklas, R.B. 2000. Dynein is a transient kinetochore component whose binding is regulated by microtubule attachment, not tension. J. Cell Biol. 151: 739748.

Kiyomitsu, T., Obuse, C., and Yanagida, M. 2007. Human Blinkin/AF15q14 is required for chromosome alignment and the mitotic checkpoint through direct interaction with Bub1 and BubR1. Dev. Cell 13: 663-676.

Kops, G.J., Kim, Y., Weaver, B.A., Mao, Y., McLeod, I., Yates, J.R., Tagaya, M., and Cleveland, D.W. 2005. ZW10 links mitotic checkpoint signaling to the structural kinetochore. $I$. Cell Biol. 169: 49-60.

Lampson, M.A. and Kapoor, T.M. 2005. The human mitotic checkpoint protein BubR1 regulates chromosome-spindle attachments. Nat. Cell Biol. 7: 93-98.

Li, Y., Yu, W., Liang, Y., and Zhu, X. 2007. Kinetochore dynein generates a poleward pulling force to facilitate congression and full chromosome alignment. Cell Res. 17: 701-712.

Martin-Lluesma, S., Stucke, V.M., and Nigg, E.A. 2002. Role of hec1 in spindle checkpoint signaling and kinetochore recruitment of $\mathrm{mad} 1 / \mathrm{mad}$. Science 297: 2267-2270.

Maure, J.F., Kitamura, E., and Tanaka, T.U. 2007. Mps1 kinase promotes sister-kinetochore bi-orientation by a tension-dependent mechanism. Curr. Biol. 17: 2175-2182.

Meraldi, P. and Sorger, P.K. 2005. A dual role for Bub1 in the spindle checkpoint and chromosome congression. EMBO J. 24: 1621-1633.

Meraldi, P., Draviam, V.M., and Sorger, P.K. 2004. Timing and checkpoints in the regulation of mitotic progression. Dev. Cell 7: 45-60.

Musacchio, A. and Salmon, E.D. 2007. The spindle-assembly checkpoint in space and time. Nat. Rev. Mol. Cell Biol. 8:
379-393.

Rieder, C.L. and Alexander, S.P. 1990. Kinetochores are transported poleward along a single astral microtubule during chromosome attachment to the spindle in newt lung cells. $J$. Cell Biol. 110: 81-95.

Ruchaud, S., Carmena, M., and Earnshaw, W.C. 2007. Chromosomal passengers: Conducting cell division. Nat. Rev. Mol. Cell Biol. 8: 798-812.

Savoian, M.S., Goldberg, M.L., and Rieder, C.L. 2000. The rate of poleward chromosome motion is attenuated in Drosophila zw10 and rod mutants. Nat. Cell Biol. 2: 948-952.

Starr, D.A., Williams, B.C., Hays, T.S., and Goldberg, M.L. 1998. ZW10 helps recruit dynactin and dynein to the kinetochore. J. Cell Biol. 142: 763-774.

Tanaka, T. and Desai, A. 2008. Kinetochore-microtubule interactions: The means to the end. Curr. Opin. Cell Biol. 20: 53-63.

Tanaka, K., Kitamura, E., Kitamura, Y., and Tanaka, T.U. 2007. Molecular mechanisms of microtubule-dependent kinetochore transport toward spindle poles. J. Cell Biol. 178: 269281.

Vorozhko, V.V., Emanuele, M.J., Kallio, M.J., Stukenberg, P.T., and Gorbsky, G.J. 2008. Multiple mechanisms of chromosome movement in vertebrate cells mediated through the Ndc80 complex and dynein/dynactin. Chromosoma 117: 169-179.

Wei, R.R., Al-Bassam, J., and Harrison, S.C. 2007. The Ndc80/ HEC1 complex is a contact point for kinetochore-microtubule attachment. Nat. Struct. Mol. Biol. 14: 54-59.

Williams, B.C., Karr, T.L., Montgomery, J.M., and Goldberg M.L. 1992. The Drosophila 1(1)zw10 gene product, required for accurate mitotic chromosome segregation, is redistributed at anaphase onset. J. Cell Biol. 118: 759-773.

Wojcik, E., Basto, R., Serr, M., Scaërou, F., Karess, R., and Hays, T. 2001. Kinetochore dynein: Its dynamics and role in the transport of the Rough deal checkpoint protein. Nat. Cell Biol. 3: 1001-1007.

Yang, Z., Tulu, U.S., Wadsworth, P., and Rieder, C.L. 2007. Kinetochore dynein is required for chromosome motion and congression independent of the spindle checkpoint. Curr. Biol. 17: 973-980. 


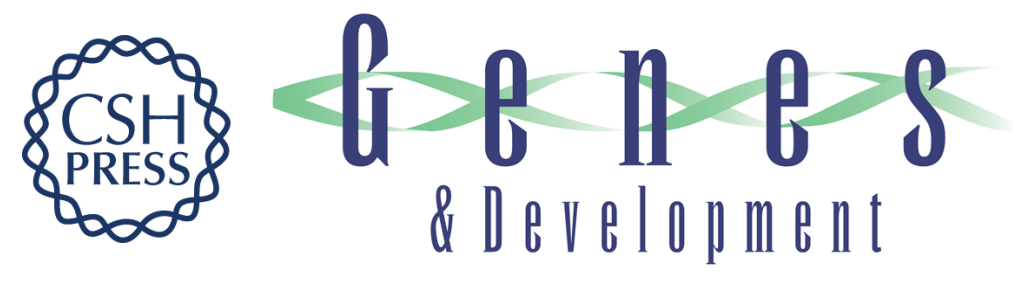

\section{Spindly attachments}

Filiz Çivril and Andrea Musacchio

Genes Dev. 2008, 22:

Access the most recent version at doi:10.1101/gad.1719208

Related Content

References

License

Email Alerting

Service
A new mechanism controlling kinetochoremicrotubule interactions revealed by comparison of two dynein-targeting components: SPDL-1 and the Rod/Zwilch/Zw10 complex

Reto Gassmann, Anthony Essex, Jia-Sheng Hu, et al.

Genes Dev. September , 2008 22: 2385-2399

This article cites 39 articles, 14 of which can be accessed free at:

http://genesdev.cshlp.org/content/22/17/2302.full.html\#ref-list-1

Articles cited in:

http://genesdev.cshlp.org/content/22/17/2302.full.html\#related-urls

Receive free email alerts when new articles cite this article - sign up in the box at the top right corner of the article or click here.

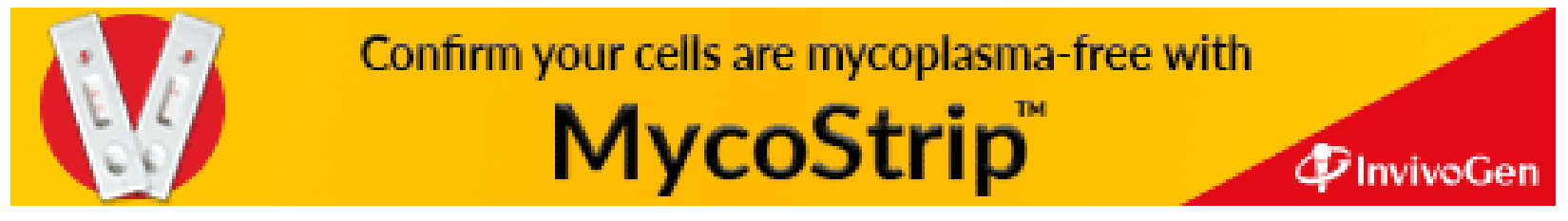

